\title{
Functionally Fitted Block Method for Solving the General Oscillatory Second-Order Initial Value Problems and Hyperbolic Partial Differential Equations
}

\author{
S. N. Jator $\mathbb{D}^{\mathbb{D}}{ }^{1}$ F. F. Ngwane $\mathbb{D}^{\mathbb{D}}{ }^{2}$ and N. O. Kirby ${ }^{1}$ \\ ${ }^{1}$ Department of Mathematics and Statistics, Austin Peay State University, Clarksville, TN 37044, USA \\ ${ }^{2}$ Department of Mathematics, University of South Carolina, Salkehatchie, Walterboro, SC 29488, USA \\ Correspondence should be addressed to S. N. Jator; jators@apsu.edu
}

Received 27 October 2018; Revised 23 January 2019; Accepted 4 February 2019; Published 26 February 2019

Academic Editor: Vincenzo Vespri

Copyright (C) 2019 S. N. Jator et al. This is an open access article distributed under the Creative Commons Attribution License, which permits unrestricted use, distribution, and reproduction in any medium, provided the original work is properly cited.

We present a block hybrid functionally fitted Runge-Kutta-Nyström method (BHFNM) which is dependent on the stepsize and a fixed frequency. Since the method is implemented in a block-by-block fashion, the method does not require starting values and predictors inherent to other predictor-corrector methods. Upon deriving our method, stability is illustrated, and it is used to numerically solve the general second-order initial value problems as well as hyperbolic partial differential equations. In doing so, we demonstrate the method's relative accuracy and efficiency.

\section{Introduction}

We consider the numerical solution of the second-order IVP

$$
\begin{aligned}
y^{\prime \prime} & =f\left(x, y, y^{\prime}\right), \\
y\left(x_{0}\right) & =y_{0}, \\
y^{\prime}\left(x_{0}\right) & =y_{0}^{\prime},
\end{aligned}
$$

$$
x \in[a, b]
$$

where $f: \mathbb{R} \times \mathbb{R}^{m} \times \mathbb{R}^{m} \longrightarrow \mathbb{R}^{m}$ is a smooth function and $m$ is the dimension of the system and its application to hyperbolic partial differential equations. Frequently, whether analyzing or numerically solving systems like (1), the system is reduced to an equivalent system of first-order IVP of dimension $2 \mathrm{~m}$ to leverage methods developed for first-order systems (cf. Lambert [1, 2], Hairer et al. [3], Hairer [4], and Brugnano et al. $[5,6]$ in the numerical setting).

There are a wide range of methods that avoid orderreduction provided they have the special form $y^{\prime \prime}=f(x, y)$. Importantly, these methods require less storage space and fewer function evaluations (cf. Hairer [4], Hairer et al. [7], Simos [8], Lambert et al., and [9], Twizell et al. [10]). Fewer methods have been developed which both avoid the orderreduction and solve the general second-order IVP (1) (see Vigo-Aguiar et al. [11], Awoyemi [12], Chawla and Sharma [13], Mahmoud and Osman [14], Franco [15], and Jator [16, 17]). Still other methods have been developed for classes of second-order IVP which use qualitative features of solutions (see Coleman and Duxbury [18], Coleman and Ixaru [19], Simos [20], Vanden et al. [21], Vigo-Aguiar et al. [11], Fang et al. [22], Nguyen et al. [23], Ramos and Vigo-Aguiar [24], Franco and Gomez [25], and Ozawa [26]).

Our objective is to present a block hybrid functionally fitted Runge-Kutta-Nyström method (BHFNM) that is implemented in a block-by-block fashion, which does not suffer from the disadvantages of requiring starting values and predictors inherent to predictor-corrector methods (see Jator et al. [27], Jator [16], and Ngwane and Jator [28]). We note that multiderivative trigonometrically fitted block methods for $y^{\prime \prime}=f\left(x, y, y^{\prime}\right)$ have been proposed in Jator $[16,29]$. However, the BHFNM proposed in this paper avoids the computation of higher-order derivatives which have the potential to increase computational cost, especially when applied to nonlinear systems. It is also the case that the proposed method performs better than those given in 
Ngwane and Jator [30, 31]. In this paper, we propose a BHFNM which is of order 5 and its application is extended to solving oscillatory systems and hyperbolic partial differential equations. It is in the same spirit as those presented by Ngwane and Jator [30, 31].

The organization of this article is as follows. In Section 2, we derive the BHFNM for solving (1). The analysis and implementation of the BHFNM are discussed in Section 3. Numerical examples are given in Section 4 to show the accuracy and efficiency of the BHFNM. Finally, the conclusion of the paper is given in Section 5.

\section{Development of the BHFNM}

To simplify the presentation we introduce the following notation: let $h>0$ denote the stepsize in the method, $x_{i}=$ $x_{0}+i h$ for any real number $i$, and

$$
J=\{r<s<v<1\}
$$

be a set of positive real numbers that are to be chosen, which will be used to specify intermediate points used in the method described below. Further, let $J_{0}=J \cup\{0\}$.

In order to numerically integrate (1), we define the BHFNM which is applied on the partition $Q_{N}=\left\{x_{n}\right.$ : $n=0,1, \ldots, N\}$, in which the step $\left[x_{n}, y_{n}, y_{n}^{\prime}\right] \longmapsto$ $\left[x_{n+1}, y_{n+1}, y_{n+1}^{\prime}\right]$ is given by combining the main methods: for each $j \in J$,

$$
y_{n+j}=\alpha_{j, 0} y_{n}+h \gamma_{j, 0} y_{n}^{\prime}+h^{2} \sum_{k \in J_{0}} \beta_{j, k} f_{n+k},
$$

and

$$
h y_{n+j}^{\prime}=h \gamma_{j, 0}^{\prime} y_{n}^{\prime}+h^{2} \sum_{k \in J_{0}} \beta_{j, k}^{\prime} f_{n+k}
$$

where $\alpha_{j, 0}, \gamma_{j, 0}, \gamma_{j, 0}^{\prime}, \beta_{j, k}, \beta_{j, k}^{\prime}$ for $j \in J$ and $k \in J_{0}$ are coefficients to be determined and $f_{n+j}=f\left(x_{n+j}, y_{n+j}, y_{n+j}^{\prime}\right)$. We note that $y_{n+j}$ is the numerical approximation to the analytical solution evaluated at $x_{n+j}$.

The coefficients of the method are chosen so that the method integrates the IVP (1) exactly whenever the solutions are members of the linear space $\left\langle 1, x, x^{2}, x^{3}, x^{4}, \sin (w x), \cos (w x)\right\rangle$, and they will depend on the frequency $w$ as well as the step-length $h$.

In order to derive the main methods and additional methods, we initially seek a continuous local approximation $\Pi(x)$ on the interval $\left[x_{n}, x_{n+1}\right]$, expressed in vector form as

$$
\Pi(x)=\left[\begin{array}{lll}
\Omega_{0}(x) & \cdots & \Omega_{6}(x)
\end{array}\right]\left[\begin{array}{c}
\ell_{0} \\
\ell_{1} \\
\vdots \\
\ell_{6}
\end{array}\right],
$$

where $L=\left[\ell_{0}, \ell_{1}, \ldots, \ell_{6}\right]^{\top}$ is a vector coefficients to be uniquely determined and $\Omega_{i}(x)=x^{i}, i=0,1,2,3,4$,
$\Omega_{5}(x)=\sin w x$, and $\Omega_{6}(x)=\cos w x$ are basis functions. In order to determine the coefficients given by $L$, we impose the following conditions on (5):

$$
\begin{aligned}
\Pi\left(x_{n}\right) & =y_{n}, \\
\Pi^{\prime}\left(x_{n}\right) & =y_{n}^{\prime}, \\
\Pi^{\prime \prime}\left(x_{n+j}\right) & =f_{n+j}, \quad j \in J_{0},
\end{aligned}
$$

and we obtain a system of equations which is expressed as

$$
\left[\begin{array}{ccc}
\Omega_{0}\left(x_{n}\right) & \cdots & \Omega_{6}\left(x_{n}\right) \\
\Omega_{0}^{\prime}\left(x_{n}\right) & \cdots & \Omega_{6}^{\prime}\left(x_{n}\right) \\
\Omega_{0}^{\prime \prime}\left(x_{n}\right) & \cdots & \Omega_{6}^{\prime \prime}\left(x_{n}\right) \\
\Omega_{0}^{\prime \prime}\left(x_{n+r}\right) & \cdots & \Omega_{6}^{\prime \prime}\left(x_{n+r}\right) \\
\Omega_{0}^{\prime \prime}\left(x_{n+s}\right) & \cdots & \Omega_{66}^{\prime \prime}\left(x_{n+s}\right) \\
\Omega_{0}^{\prime \prime}\left(x_{n+v}\right) & \cdots & \Omega_{6}^{\prime \prime}\left(x_{n+v}\right) \\
\Omega_{0}^{\prime \prime}\left(x_{n+1}\right) & \cdots & \Omega_{6}^{\prime \prime}\left(x_{n+1}\right)
\end{array}\right]\left[\begin{array}{l}
\ell_{0} \\
\ell_{1} \\
\ell_{2} \\
\ell_{3} \\
\ell_{4} \\
\ell_{5} \\
\ell_{6}
\end{array}\right]=\left[\begin{array}{c}
y_{n} \\
y_{n}^{\prime} \\
f_{n} \\
f_{n+r} \\
f_{n+s} \\
f_{n+v} \\
f_{n+1}
\end{array}\right]
$$

We note that $y_{n+j}=\Pi\left(x_{n+j}\right)$ is the numerical approximation to the analytical solution $y\left(x_{n+j}\right), y_{n+j}^{\prime}=\Pi^{\prime}\left(x_{n+j}\right)$ is the numerical approximation to $y^{\prime}\left(x_{n+j}\right)$, and $f_{n+j}=\Pi^{\prime \prime}\left(x_{n+j}\right)$ is an approximation to $y^{\prime \prime}\left(x_{n+j}\right)$, for $j \in J_{0}$.

System (7) is solved with the aid of Mathematica to obtain the coefficients given by $L$, which are substituted into (5) to give the continuous scheme given by

$\Pi(x)$

$$
=\left[\begin{array}{lll}
\Omega_{0}(x) \cdots & \Omega_{6}(x)
\end{array}\right]\left[\begin{array}{ccc}
\Omega_{0}\left(x_{n}\right) & \cdots & \Omega_{6}\left(x_{n}\right) \\
\Omega_{0}^{\prime}\left(x_{n}\right) & \cdots & \Omega_{6}^{\prime}\left(x_{n}\right) \\
\Omega_{0}^{\prime \prime}\left(x_{n}\right) & \cdots & \Omega_{6}^{\prime \prime}\left(x_{n}\right) \\
\Omega_{0}^{\prime \prime}\left(x_{n+r}\right) & \cdots & \Omega_{6}^{\prime \prime}\left(x_{n+r}\right) \\
\Omega_{0}^{\prime \prime}\left(x_{n+s}\right) & \cdots & \Omega_{66}^{\prime \prime}\left(x_{n+s}\right) \\
\Omega_{0}^{\prime \prime}\left(x_{n+v}\right) & \cdots & \Omega_{6}^{\prime \prime}\left(x_{n+v}\right) \\
\Omega_{0}^{\prime \prime}\left(x_{n+1}\right) & \cdots & \Omega_{6}^{\prime \prime}\left(x_{n+1}\right)
\end{array}\right]^{-1}\left[\begin{array}{c}
y_{n} \\
y_{n}^{\prime} \\
f_{n} \\
f_{n+r} \\
f_{n+s} \\
f_{n+v} \\
f_{n+1}
\end{array}\right]
$$

which after simplification takes the form

$$
\Pi(x)=\alpha_{0}(x) y_{n}+\delta_{0}(x) h y_{n}^{\prime}+h^{2} \sum_{j \in J_{0}} \beta_{j}(x) f_{n+j},
$$

where $\alpha_{0}(x), \delta_{0}(x)$, and $\beta_{j}(x)$ for $j \in J_{0}$ are continuous coefficients. The first derivative of (9) is given by

$$
\Pi^{\prime}(x)=\frac{d}{d x} \Pi(x) .
$$

Remark 1. We note that in the derivation of the BHFNM, the basis functions $\Omega_{i}(x)=x^{i}, i=0,1,2,3,4, \Omega_{5}(x)=\sin w x$, and $\Omega_{6}(x)=\cos w x$ are chosen because they are simple to analyze. Nevertheless, other possible bases are possible (see Nguyen et al. [23] and Hoang et al. [23]). We note that the zeros of the Chebyshev's polynomial of the first kind or the zeros of the Legendre's polynomial can also be chosen. However, we elect not to use them in this case because upon testing them the results were found to be the same as for the chosen points. 
2.1. Specification of the Method. The continuous form (8) and its first derivative, which are equivalent to the forms (9) and (10), are used to generate four discrete methods and four additional methods. The discrete and additional methods are then applied as a BHFNM for solving (1). We choose $J=$ $\{r, s, v, 1\}=\{1 / 4,1 / 2,3 / 4,1\}$, and evaluating (9) at $x=x_{n+j}$, $j \in J$, gives the four discrete methods $y_{n+j}=\Pi\left(x_{n}+j h\right)$, which takes the form of the main methods (3). Evaluating (10) at $x=x_{n+j}$ gives the additional methods $y_{n+j}^{\prime}=\Pi^{\prime}\left(x_{n}+\right.$ $j h)$, with $j \in J$, which takes the form of the additional methods (4). The coefficients and the corresponding Taylor series equivalence of $y_{n+j}, h y_{n+j}^{\prime}$ where $j \in J$ are, respectively, given in terms of $u=w h$ as follows:

$$
\begin{aligned}
& \alpha_{r, 0}=1 \text {, } \\
& \gamma_{r, 0}=\frac{1}{2} \\
& \beta_{r, 0}=\frac{\csc ^{4}(u / 8) \sec (u / 8)\left[5 u^{2} \cos (u / 8)-\left(9 u^{2}+128\right) \cos (3 u / 8)+32(u \sin (5 u / 8)+4 \cos (5 u / 8))\right]}{2048 u^{2}} \\
& =\frac{367}{23040}+\frac{199 u^{2}}{6193152}+\frac{1543 u^{4}}{14863564800}+\frac{281 u^{6}}{597939978240}+\frac{7261781 u^{8}}{2742391916199936000}+\frac{31117 u^{10}}{1914688174219591680}+\ldots \\
& \beta_{r, r}=\frac{3\left(9 u^{2}-4 u \tan (u / 8)-384\right)+\left(672-22 u^{2}\right) \csc ^{2}(u / 8)+276 u \cot (u / 8)+3 u(u-18 \sin (u / 4)) \csc ^{4}(u / 8)}{384 u^{2}} \\
& =\frac{3}{128}-\frac{337 u^{2}}{3870720}-\frac{457 u^{4}}{1857945600}-\frac{289 u^{6}}{290665267200}-\frac{166697 u^{8}}{31163544502272000}-\frac{2138501 u^{10}}{65817405988798464000}+\ldots \\
& \beta_{r, s}=\frac{\csc ^{4}(u / 8)\left[\left(576-11 u^{2}\right) \cos (u / 2)-\left(7 u^{2}+576\right) \cos (u / 4)+144 u \sin (u / 2)\right]}{1536 u^{2}} \\
& =-\frac{47}{3840}+\frac{353 u^{2}}{5160960}+\frac{19 u^{4}}{165150720}+\frac{569 u^{6}}{3487983206400}+\frac{72887 u^{8}}{457065319366656000}-\frac{151 u^{10}}{4178882919923712000}+\ldots \\
& \beta_{r, v}=\frac{7 u^{2}+480 \csc ^{2}(u / 8)+3 u \csc ^{4}(u / 8) \sec (u / 8)[u \cos (3 u / 8)-2(3 \sin (3 u / 8)+\sin (5 u / 8))]-384}{384 u^{2}} \\
& =\frac{29}{5760}-\frac{u^{2}}{241920}+\frac{43 u^{4}}{464486400}+\frac{127 u^{6}}{163499212800}+\frac{12577 u^{8}}{2448564210892800}+\frac{20593 u^{10}}{632859672969216000}+\ldots \\
& \beta_{r, 1}=\frac{\csc ^{4}(u / 8)\left[u^{2}+\left(384-7 u^{2}\right) \cos (u / 4)+48 u \sin (3 u / 8) \sec (u / 8)-384\right]}{3072 u^{2}} \\
& =-\frac{7}{7680}-\frac{289 u^{2}}{30965760}-\frac{139 u^{4}}{2123366400}-\frac{2899 u^{6}}{6975966412800}-\frac{7116007 u^{8}}{2742391916199936000}-\frac{8563517 u^{10}}{526539247910387712000} \\
& +\ldots \\
& \alpha_{s, 0}=1 \text {, } \\
& \gamma_{s, 0}=\frac{1}{2} \\
& \beta_{s, 0}=\frac{\csc ^{4}(u / 8)\left[7 u^{2}-10 u^{2} \cos (u / 4)+12 \sec (u / 8)(u \sin (5 u / 8)+2 \cos (5 u / 8))-24\right]}{384 u^{2}} \\
& =\frac{53}{1440}+\frac{19 u^{2}}{241920}+\frac{37 u^{4}}{154828800}+\frac{163 u^{6}}{163499212800}+\frac{461287 u^{8}}{85699747381248000}+\frac{12773 u^{10}}{391770273742848000}+\ldots
\end{aligned}
$$




$$
\begin{aligned}
& \beta_{\mathrm{s}, r}=\frac{20 u^{2}+3 u^{2} \csc ^{4}(u / 8)-16\left(u^{2}-15\right) \csc ^{2}(u / 8)-6 u \tan (u / 8)+6 u \cot (u / 8)\left(23-9 \csc ^{2}(u / 8)\right)-288}{96 u^{2}} \\
& =\frac{1}{10}-\frac{13 u^{2}}{60480}-\frac{67 u^{4}}{116121600}-\frac{29 u^{6}}{13624934400}-\frac{46559 u^{8}}{4284987369062400}-\frac{26753 u^{10}}{411358787429990400}+\ldots \\
& \beta_{s, s}=-\frac{\csc ^{4}(u / 8)\left[2 u^{2} \cos (u / 4)+\left(u^{2}-24\right) \cos (u / 2)-12 u \sin (u / 2)+24\right]}{64 u^{2}} \\
& =-\frac{1}{48}+\frac{u^{2}}{5760}+\frac{23 u^{4}}{77414400}+\frac{u^{6}}{2477260800}+\frac{331 u^{8}}{1098714710016000}-\frac{703 u^{10}}{1371195958099968000}+\ldots \\
& \beta_{s, v}=\frac{\left(3 \csc ^{2}(u / 8)-2\right)\left(-2 u^{2}+u^{2} \csc ^{2}(u / 8)+48\right)+6 u \tan (u / 8)+6 u \cot (u / 8)\left(9-7 \csc ^{2}(u / 8)\right)}{96 u^{2}} \\
& =\frac{1}{90}-\frac{u^{2}}{60480}+\frac{u^{4}}{5529600}+\frac{13 u^{6}}{8174960640}+\frac{32027 u^{8}}{3060705263616000}+\frac{15019 u^{10}}{228532659683328000}+\ldots \\
& \beta_{s, 1}=-\frac{\csc ^{4}(u / 8)\left[u^{2}+2\left(u^{2}-24\right) \cos (u / 4)-12 u \sin (3 u / 8) \sec (u / 8)+48\right]}{384 u^{2}} \\
& =-\frac{1}{480}-\frac{u^{2}}{48384}-\frac{13 u^{4}}{92897280}-\frac{47 u^{6}}{54499737600}-\frac{452681 u^{8}}{85699747381248000}-\frac{269639 u^{10}}{8227175748599808000}+\ldots \\
& \alpha_{v, 0}=1 \text {, } \\
& \gamma_{v, 0}=\frac{3}{4} \\
& \beta_{v, 0}=\frac{\csc ^{4}(u / 8)\left[27 u^{2}-45 u^{2} \cos (u / 4)+16 \sec (u / 8)(3 u \sin (5 u / 8)+4 \cos (5 u / 8))-64\right]}{1024 u^{2}} \\
& =\frac{147}{2560}+\frac{141 u^{2}}{1146880}+\frac{67 u^{4}}{183500800}+\frac{1157 u^{6}}{775107379200}+\frac{8233 u^{8}}{1025960312832000}+\frac{316627 u^{10}}{6500484542103552000}+\ldots \\
& \beta_{v, r}=\frac{3\left\{15 u^{2}+96 \csc ^{2}(u / 8)+u \csc ^{4}(u / 8) \sec (u / 8)[3 u \cos (3 u / 8)-2(\sin (3 u / 8)+3 \sin (5 u / 8))]-128\right\}}{128 u^{2}} \\
& =\frac{117}{640}-\frac{3 u^{2}}{8960}-\frac{u^{4}}{1146880}-\frac{19 u^{6}}{6055526400}-\frac{5647 u^{8}}{352673857536000}-\frac{9811 u^{10}}{101570070970368000}+\ldots \\
& \beta_{v, s}=-\frac{3 \csc ^{4}(u / 8)\left[\left(3 u^{2}-64\right) \cos (u / 2)+\left(15 u^{2}+64\right) \cos (u / 4)-48 u \sin (u / 2)\right]}{512 u^{2}} \\
& =\frac{27}{1280}+\frac{153 u^{2}}{573440}+\frac{39 u^{4}}{91750400}+\frac{59 u^{6}}{129184563200}-\frac{211 u^{8}}{1880927240192000}-\frac{107 u^{10}}{43336563614023680}+\ldots \\
& \beta_{v, v}=\frac{9 u^{2}+9 u^{2} \csc ^{4}(u / 8)+\left(96-18 u^{2}\right) \csc ^{2}(u / 8)+12 u \tan (u / 8)+12 u \cot (u / 8)\left(9-7 \csc ^{2}(u / 8)\right)-128}{128 u^{2}} \\
& =\frac{3}{128}-\frac{3 u^{2}}{143360}+\frac{u^{4}}{3276800}+\frac{7 u^{6}}{2768240640}+\frac{3257 u^{8}}{201527918592000}+\frac{81163 u^{10}}{812560567762944000}+\ldots \\
& \beta_{v, 1}=\frac{3 \csc ^{4}(u / 8) \sec (u / 8)\left(8 \sin (3 u / 8)-3 u \cos ^{3}(u / 8)\right)}{512 u} \\
& =-\frac{9}{2560}-\frac{39 u^{2}}{1146880}-\frac{41 u^{4}}{183500800}-\frac{1039 u^{6}}{775107379200}-\frac{18197 u^{8}}{2257112688230400}-\frac{321977 u^{10}}{6500484542103552000}+\ldots
\end{aligned}
$$




$$
\begin{aligned}
& \alpha_{1,0}=1, \\
& \gamma_{1,0}=1 \\
& \beta_{1,0}=\frac{\csc ^{4}(u / 8) \sec (u / 8)\left[2(u \sin (5 u / 8)+\cos (5 u / 8))-\left(u^{2}+2\right) \cos (3 u / 8)\right]}{32 u^{2}} \\
& =\frac{7}{90}+\frac{u^{2}}{6048}+\frac{u^{4}}{2073600}+\frac{u^{6}}{510935040}+\frac{28319 u^{8}}{2678117105664000}+\frac{83 u^{10}}{1285496210718720}+\ldots \\
& \beta_{1, r} \\
& =\frac{\csc ^{4}(u / 8) \sec (u / 8)\left[8 u^{2} \cos (u / 8)+\left(u^{2}+12\right) \cos (3 u / 8)+3\left(u^{2}-4\right) \cos (5 u / 8)-6 u \sin (3 u / 8)-18 u \sin (5 u / 8)\right]}{96 u^{2}} \\
& =\frac{4}{15}-\frac{u^{2}}{2160}-\frac{17 u^{4}}{14515200}-\frac{u^{6}}{243302400}-\frac{56153 u^{8}}{2678117105664000}-\frac{32783 u^{10}}{257099242143744000}+\ldots \\
& \beta_{1, s}=-\frac{\csc ^{4}(u / 8)(-18 \sin (u / 2)+u \cos (u / 2)+8 u \cos (u / 4))}{48 u} \\
& =\frac{1}{15}+\frac{u^{2}}{2520}+\frac{u^{4}}{1612800}+\frac{u^{6}}{1703116800}-\frac{97 u^{8}}{178541140377600}-\frac{139 u^{10}}{28566582460416000}+\ldots \\
& \beta_{1, v}=\frac{4 u^{2}+3 u^{2} \csc ^{4}(u / 8)-6\left(u^{2}+4\right) \csc ^{2}(u / 8)+3 u \tan (u / 8)+3 u \cot (u / 8)\left(9-7 \csc ^{2}(u / 8)\right)+48}{24 u^{2}} \\
& =\frac{4}{45}-\frac{u^{2}}{15120}+\frac{u^{4}}{2903040}+\frac{17 u^{6}}{5109350400}+\frac{8299 u^{8}}{382588157952000}+\frac{34451 u^{10}}{257099242143744000}+\ldots \\
& \beta_{1,1}=-\frac{\csc ^{4}(u / 8) \sec (u / 8)\left[2 u^{2} \cos (u / 8)+\left(u^{2}-6\right) \cos (3 u / 8)-6 u \sin (3 u / 8)+6 \cos (5 u / 8)\right]}{96 u^{2}} \\
& =-\frac{u^{2}}{30240}-\frac{u^{4}}{3628800}-\frac{u^{6}}{567705600}-\frac{7201 u^{8}}{669529276416000}-\frac{17 u^{10}}{256842399744000}+\ldots \\
& \gamma_{r, 0}^{\prime}=1 \\
& \beta_{r, 0}^{\prime}=\frac{\csc ^{4}(u / 8) \sec (u / 8)[48(\sin (5 u / 8)-\sin (3 u / 8))+11 u \cos (u / 8)-23 u \cos (3 u / 8)]}{768 u} \\
& =\frac{251}{2880}+\frac{863 u^{2}}{3870720}+\frac{71 u^{4}}{88473600}+\frac{1409 u^{6}}{373712486400}+\frac{1461541 u^{8}}{68559797904998400}+\frac{408887 u^{10}}{3134162189942784000}+\ldots \\
& \beta_{r, r}^{\prime}=\frac{-24 \tan (u / 8)+u\left(6 \csc ^{4}(u / 8)-37 \csc ^{2}(u / 8)+46\right)+\cot (u / 8)\left(264-48 \csc ^{2}(u / 8)\right)}{96 u} \\
& =\frac{323}{1440}-\frac{1159 u^{2}}{1935360}-\frac{589 u^{4}}{309657600}-\frac{10519 u^{6}}{1307993702400}-\frac{135067 u^{8}}{3116354450227200}-\frac{2869897 u^{10}}{10969567664799744000}+\ldots \\
& \beta_{r, s}^{\prime}=-\frac{\csc ^{4}(u / 8)[5 u \cos (u / 4)+4(-9 \sin (u / 2)+9 \sin (u / 4)+u \cos (u / 2))]}{96 u} \\
& =-\frac{11}{120}+\frac{37 u^{2}}{80640}+\frac{23 u^{4}}{25804800}+\frac{41 u^{6}}{27249868800}+\frac{6049 u^{8}}{2856658246041600}+\frac{961 u^{10}}{457065319366656000}+\ldots \\
& \beta_{r, v}^{\prime}=\frac{10 u+24 \tan (u / 8)-19 u \csc ^{2}(u / 8)+3[2(u+\sin (u / 4))-5 \sin (u / 2)] \csc ^{4}(u / 8)}{96 u} \\
& =\frac{53}{1440}-\frac{5 u^{2}}{387072}+\frac{221 u^{4}}{309657600}+\frac{1579 u^{6}}{261598740480}+\frac{1388953 u^{8}}{34279898952499200}+\frac{567829 u^{10}}{2193913532959948800}+\ldots \\
& \beta_{r, 1}^{\prime}=-\frac{\csc ^{4}(u / 8) \sec (u / 8)[48(\sin (u / 8)-\sin (3 u / 8))+7 u \cos (u / 8)+5 u \cos (3 u / 8)]}{768 u} \\
& =-\frac{19}{2880}-\frac{271 u^{2}}{3870720}-\frac{313 u^{4}}{619315200}-\frac{8551 u^{6}}{2615987404800}-\frac{1413149 u^{8}}{68559797904998400}-\frac{258803 u^{10}}{1994466848145408000}+\ldots
\end{aligned}
$$




$$
\begin{aligned}
& \gamma_{s, 0}^{\prime}=1 \\
& \beta_{s, 0}^{\prime}=\frac{\csc ^{4}(u / 8)[4(u+3 \sin (u / 2)-3 \sin (u / 4))-7 u \cos (u / 4)]}{96 u} \\
& =\frac{29}{360}+\frac{37 u^{2}}{241920}+\frac{23 u^{4}}{77414400}+\frac{41 u^{6}}{81749606400}+\frac{6049 u^{8}}{8569974738124800}+\frac{961 u^{10}}{1371195958099968000}+\ldots \\
& \beta_{s, r}^{\prime}=\frac{\csc ^{4}(u / 8)(11 u-36 \sin (u / 2)+24 \sin (u / 4)+7 u \cos (u / 2)-6 u \cos (u / 4))}{96 u} \\
& =\frac{31}{90}-\frac{53 u^{2}}{120960}-\frac{31 u^{4}}{38707200}-\frac{7 u^{6}}{5839257600}-\frac{5273 u^{8}}{4284987369062400}+\frac{151 u^{10}}{685597979049984000}+\ldots \\
& \beta_{s, s}^{\prime}=-\frac{\csc ^{4}(u / 8)(-18 \sin (u / 2)+u \cos (u / 2)+8 u \cos (u / 4))}{48 u} \\
& =\frac{1}{15}+\frac{u^{2}}{2520}+\frac{u^{4}}{1612800}+\frac{u^{6}}{1703116800}-\frac{97 u^{8}}{178541140377600}-\frac{139 u^{10}}{28566582460416000}+\ldots \\
& \beta_{s, v}^{\prime}=\frac{\cot ^{2}(u / 8) \csc ^{2}(u / 8)[u(\cos (u / 4)+2)-12 \sin (u / 4)]}{24 u} \\
& =\frac{1}{90}-\frac{11 u^{2}}{120960}-\frac{u^{4}}{38707200}+\frac{17 u^{6}}{40874803200}+\frac{8377 u^{8}}{4284987369062400}+\frac{4297 u^{10}}{685597979049984000}+\ldots \\
& \beta_{s, 1}^{\prime}=-\frac{\csc ^{4}(u / 8)[u(\cos (u / 4)+2)-12 \sin (u / 4)]}{96 u} \\
& =-\frac{1}{360}-\frac{u^{2}}{48384}-\frac{u^{4}}{11059200}-\frac{u^{6}}{3269984256}-\frac{691 u^{8}}{779088612556800}-\frac{u^{10}}{430516784332800}+\ldots \\
& \gamma_{v, 0}^{\prime}=1 \\
& \beta_{v, 0}^{\prime}=\frac{\csc ^{4}(u / 8) \sec (u / 8)[16(\sin (u / 8)+\sin (5 u / 8))-3 u(\cos (u / 8)+3 \cos (3 u / 8))]}{256 u} \\
& =\frac{27}{320}+\frac{29 u^{2}}{143360}+\frac{7 u^{4}}{9830400}+\frac{1007 u^{6}}{290665267200}+\frac{17293 u^{8}}{846417258086400}+\frac{44623 u^{10}}{348240243326976000}+\ldots \\
& \beta_{v, r}^{\prime}=\frac{-8 \tan (u / 8)+3 u\left(2 \csc ^{4}(u / 8)-5 \csc ^{2}(u / 8)+6\right)+\cot (u / 8)\left(88-48 \csc ^{2}(u / 8)\right)}{32 u} \\
& =\frac{51}{160}-\frac{37 u^{2}}{71680}-\frac{53 u^{4}}{34406400}-\frac{991 u^{6}}{145332633600}-\frac{1531 u^{8}}{38473511731200}-\frac{307553 u^{10}}{1218840851644416000}+\ldots \\
& \beta_{v, s}^{\prime}=\frac{3 \csc ^{4}(u / 8)[4(\sin (u / 2)+\sin (u / 4))-3 u \cos (u / 4)]}{32 u} \\
& =\frac{9}{40}+\frac{3 u^{2}}{8960}+\frac{u^{4}}{2867200}-\frac{u^{6}}{3027763200}-\frac{113 u^{8}}{35267385753600}-\frac{601 u^{10}}{50785035485184000}+\ldots \\
& \beta_{v, v}^{\prime}=\frac{6 u+8 \tan (u / 8)+6 u \csc ^{4}(u / 8)-9 u \csc ^{2}(u / 8)+8 \cot (u / 8)\left(5-6 \csc ^{2}(u / 8)\right)}{32 u} \\
& =\frac{21}{160}+\frac{u^{2}}{14336}+\frac{37 u^{4}}{34406400}+\frac{211 u^{6}}{29066526720}+\frac{18649 u^{8}}{423208629043200}+\frac{65357 u^{10}}{243768170328883200}+\ldots \\
& \beta_{v, 1}^{\prime}=\frac{\csc ^{4}(u / 8)[32 \sin (3 u / 8) \sec (u / 8)-6 u(\cos (u / 4)+1)]}{256 u} \\
& =-\frac{3}{320}-\frac{13 u^{2}}{143360}-\frac{41 u^{4}}{68812800}-\frac{1039 u^{6}}{290665267200}-\frac{18197 u^{8}}{846417258086400}-\frac{321977 u^{10}}{2437681703288832000}+\ldots
\end{aligned}
$$




$$
\begin{aligned}
& \gamma_{1,0}^{\prime}=1 \\
& \beta_{1,0}^{\prime}=\frac{\csc ^{4}(u / 8)(u+6 \sin (u / 2)-4 u \cos (u / 4))}{48 u} \\
& =\frac{7}{90}+\frac{u^{2}}{7560}+\frac{u^{4}}{4838400}+\frac{u^{6}}{5109350400}-\frac{97 u^{8}}{535623421132800}-\frac{139 u^{10}}{85699747381248000}+\ldots \\
& \beta_{1, r}^{\prime}=\frac{\csc ^{4}(u / 8)[u(\cos (u / 2)+2)-6 \sin (u / 2)]}{12 u} \\
& =\frac{16}{45}-\frac{u^{2}}{1890}-\frac{u^{4}}{1209600}-\frac{u^{6}}{1277337600}+\frac{97 u^{8}}{133905855283200}+\frac{139 u^{10}}{21424936845312000}+\ldots \\
& \beta_{1, s}^{\prime}=-\frac{\csc ^{4}(u / 8)(-18 \sin (u / 2)+u \cos (u / 2)+8 u \cos (u / 4))}{24 u} \\
& =\frac{2}{15}+\frac{u^{2}}{1260}+\frac{u^{4}}{806400}+\frac{u^{6}}{851558400}-\frac{97 u^{8}}{89270570188800}-\frac{139 u^{10}}{14283291230208000}+\ldots \\
& \beta_{1, v}^{\prime}=\frac{\csc ^{4}(u / 8)[u(\cos (u / 2)+2)-6 \sin (u / 2)]}{12 u} \\
& =\frac{16}{45}-\frac{u^{2}}{1890}-\frac{u^{4}}{1209600}-\frac{u^{6}}{1277337600}+\frac{97 u^{8}}{133905855283200}+\frac{139 u^{10}}{21424936845312000}+\ldots \\
& \beta_{1,1}^{\prime}=\frac{\csc ^{4}(u / 8)(u+6 \sin (u / 2)-4 u \cos (u / 4))}{48 u} \\
& =\frac{7}{90}+\frac{u^{2}}{7560}+\frac{u^{4}}{4838400}+\frac{u^{6}}{5109350400}-\frac{97 u^{8}}{535623421132800}-\frac{139 u^{10}}{85699747381248000}+\ldots
\end{aligned}
$$

Remark 2. We note that the Taylor series expansions in (11) through (18) must be used when $u \longrightarrow 0$ because the corresponding trigonometric coefficients given in these equations are vulnerable to heavy cancelations (see [8]).

\section{Properties of the Method}

3.1. Local Truncation Error. We define the local truncation errors (LTEs) of (3) and (4) specified by the coefficients in (11) through (18) as

$$
\begin{aligned}
& L_{J}\left[y\left(x_{n}\right) ; h\right]=y\left(x_{n}+j h\right)-\left(\alpha_{j, 0} y\left(x_{n}\right)\right. \\
& \left.+h \gamma_{j, 0} y^{\prime}\left(x_{n}\right)+h^{2} \sum_{k \in J_{0}} \beta_{j, k} y^{\prime \prime}\left(x_{n}+k h\right)\right), \\
& L_{J}^{\prime}\left[y\left(x_{n}\right) ; h\right]=h y^{\prime}\left(x_{n}+j h\right)-\left(h \gamma_{j, 0}^{\prime} y^{\prime}\left(x_{n}\right)\right. \\
& \left.+h^{2} \sum_{k \in J_{0}} \beta_{j, k}^{\prime} y^{\prime \prime}\left(x_{n}+k h\right)\right) .
\end{aligned}
$$

Assuming that $y(x)$ is sufficiently differentiable, we can expand the terms in $L_{J}$ as a Taylor series about the point $x_{n}$ to obtain the expressions for the LTEs $L_{J}$ and $L_{J}^{\prime}$ for $J=$ $\{r, s, v, 1\}=\{1 / 4,1 / 2,3 / 4,1\}$ as

$$
\begin{aligned}
& L_{J}\left[y\left(x_{n}\right) ; h\right] \\
& =\left\{\begin{array}{l}
\frac{107 h^{7}}{165150720}\left(w^{2} y^{(5)}\left(x_{n}\right)+y^{(7)}\left(x_{n}\right)\right)+O\left(h^{8}\right), \\
\frac{h^{7}}{645120}\left(w^{2} y^{(5)}\left(x_{n}\right)+y^{(7)}\left(x_{n}\right)\right)+O\left(h^{8}\right), \\
\frac{9 h^{7}}{3670016}\left(w^{2} y^{(5)}\left(x_{n}\right)+y^{(7)}\left(x_{n}\right)\right)+O\left(h^{8}\right), \\
\frac{h^{7}}{322560}\left(w^{2} y^{(5)}\left(x_{n}\right)+y^{(7)}\left(x_{n}\right)\right)+O\left(h^{8}\right),
\end{array}\right. \\
& L_{J}^{\prime}\left[\begin{array}{l}
\left.y\left(x_{n}\right) ; h\right] \\
\frac{3 h^{7}}{655360}\left(w^{2} y^{(5)}\left(x_{n}\right)+y^{(7)}\left(x_{n}\right)\right)+O\left(h^{8}\right), \\
\frac{h^{7}}{368640}\left(w^{2} y^{(5)}\left(x_{n}\right)+y^{(7)}\left(x_{n}\right)\right)+O\left(h^{8}\right), \\
\frac{3 h^{7}}{655360}\left(w^{2} y^{(5)}\left(x_{n}\right)+y^{(7)}\left(x_{n}\right)\right)+O\left(h^{8}\right), \\
-\frac{h^{8}}{1935360}\left(w^{2} y^{(6)}\left(x_{n}\right)+y^{(8)}\left(x_{n}\right)\right)+O\left(h^{9}\right) .
\end{array}\right.
\end{aligned}
$$

Remark 3. The local truncation error constants of the BHFNM formulated from (2) and (3) and specified by the coefficients in (11) through (18) are given, respectively, by (107/165150720, 1/645120, 9/3670016, 1/322560, 3/655360, 
$1 / 368640,3 / 655360,0)^{\top}$ and orders $(5,5,5,5,5,5,5,6)^{T}$; hence the BHFNM has order at least 5.

3.2. Block Form. BHFNM is formulated from the four discrete hybrid formulas stated in (3) which are provided by the continuous one-step hybrid trigonometrically fitted method with three off-grid points given by (9) and its first derivative (10). We define the following vectors:

$$
\begin{aligned}
& Y_{n+1} \\
& \quad=\left[y_{n+r}, y_{n+s}, y_{n+v}, y_{n+1}, h y_{n+r}^{\prime}, h y_{n+s}^{\prime}, h y_{n+v}^{\prime}, h y_{n+1}^{\prime}\right]^{\top}, \\
& Y_{n}=\left[y_{n-v}, y_{n-s}, y_{n-r}, y_{n}, h y_{n-v}^{\prime}, h y_{n-s}^{\prime}, h y_{n-r}^{\prime}, h y_{n}^{\prime}\right]^{\top}, \\
& F_{n+1} \\
& =\left[f_{n+r}, f_{n+s}, f_{n+v}, f_{n+1}, h f_{n+r}^{\prime}, h f_{n+s}^{\prime}, h f_{n+v}^{\prime}, h f_{n+1}^{\prime}\right]^{\top}, \\
& F_{n}=\left[f_{n-v}, f_{n-s}, f_{n-r}, f_{n}, h f_{n-v}^{\prime}, h f_{n-s}^{\prime}, h f_{n-r}^{\prime}, h f_{n-}^{\prime}\right]^{\top},
\end{aligned}
$$

where $n=0, \ldots, N$. The methods in (3) specified by coefficients (11)-(18) are combined to give the BHFNM, which is expressed as

$$
A_{1} Y_{n+1}=A_{0} Y_{n}+h^{2}\left(B_{0} F_{n}+B_{1} F_{n+1}\right)
$$

where $A_{0}, A_{1}, B_{0}$, and $B_{1}$ are square matrices of dimension eight whose elements characterize the method and are given by the coefficients of (3).

3.3. Stability. The linear-stability of the BHFNM is discussed by applying the method to the test equation $y^{\prime \prime}=-\lambda^{2} y$, where $\lambda$ is a real constant (see [18]). Letting $\Upsilon=\lambda h$, it is easily shown as in [19] that the application of (23) to the test equation yields

$$
\begin{aligned}
Y_{n+1} & =M\left(\Upsilon^{2} ; u\right) Y_{n}, \\
M\left(\Upsilon^{2} ; u\right) & :=\left(A_{1}-\Upsilon^{2} B_{1}\right)^{-1}\left(A_{0}+\Upsilon^{2} B_{0}\right),
\end{aligned}
$$

where the matrix $M\left(\Upsilon^{2} ; u\right)$ is the amplification matrix which determines the stability of the method.

Definition 4. A region of stability is a region in the $(q, u)$ plane, throughout which the spectral radius $\rho\left(M\left(\Upsilon^{2} ; u\right)\right) \leq 1$ and any closed curve given by $\rho\left(M\left(\Upsilon^{2} ; u\right)\right)=1$ define the stability boundary of the method (see [22]). We note that the plot for the stability region of the BHFNM method is given in Figure 1.

Remark 5. It is observed that in the $(\Upsilon, u)$-plane the BHFNM is stable for $\Upsilon \in[0,75.09]$ and $u \in[-\pi, \pi]$ (see Figure 1 ).

3.4. Implementation. The methods given by (11)-(18) are combined to form the block method BHFNM (23), which is used to solve (1) without requiring starting values and predictors. BHFNM is implemented in a block-by-block fashion using a

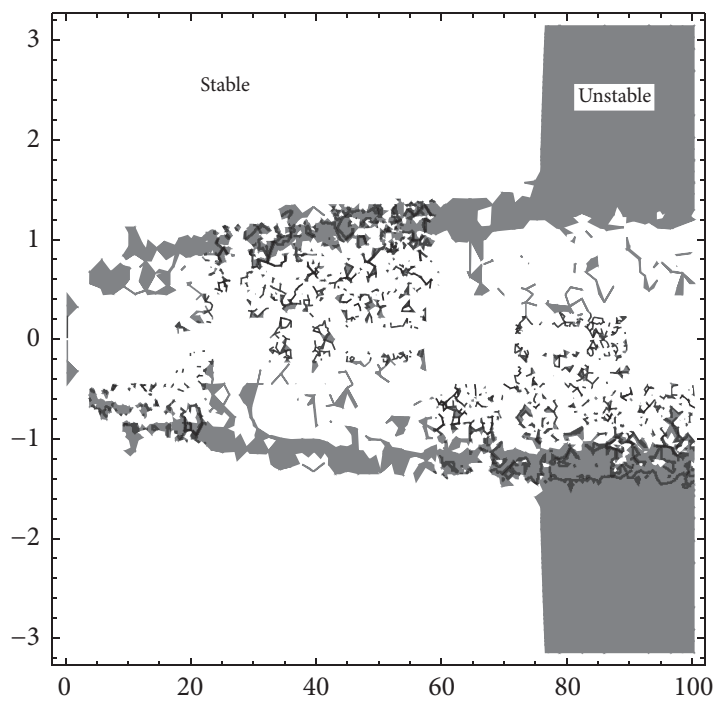

FIgURE 1: The stability region plotted in the $(\Upsilon, u)$-plane.

Mathematica 10.0 code, enhanced by the features NSolve[ ] and FindRoot [ ] (see Keiper and Gear [32]) for linear and nonlinear problems, respectively. In particular, the BHFNM is applied over $\left[x_{0}, x_{1}\right],\left[x_{1}, x_{2}\right], \ldots,\left[x_{N-1}, x_{N}\right]$ by choosing $N, h=(b-a) / N$, and using (23) to simultaneously obtain the values of

$$
\begin{array}{r}
\left(y_{n+1 / 4}, y_{n+1 / 2}, y_{n+3 / 4}, y_{n+1}, y_{n+1 / 4}^{\prime}, y_{n+1 / 2}^{\prime}, y_{n+3 / 4}^{\prime}, y_{n+1}^{\prime}\right)^{\top}, \\
n=0,1 \ldots, N-1 .
\end{array}
$$

\section{Numerical Examples}

The comparison of the following methods are given in this section:

(i) Block Nyström method of order 5 based on polynomial basis and given in [33].

(ii) Block Hybrid Trigonometrically Fitted Algorithm of order 5 given in [30].

(iii) Block hybrid trigonometrically fitted Runge-KuttaNyström method of order 3 given in [31].

(iv) Block hybrid functionally fitted Runge-KuttaNyström method (BHFNM) of order 5 given in this paper.

Example 6. We consider the following general second-order IVP given in [34]

$$
\begin{aligned}
y^{\prime \prime}+\omega^{2} y & =-\delta y^{\prime}, \\
y(0) & =1, \\
y^{\prime}(0) & =-\frac{\delta}{2},
\end{aligned}
$$


TABLE 1: Comparison of global errors for Example 6 on $[0,100], w=$ 1.

\begin{tabular}{lcccc}
\hline $\mathrm{h}$ & BHFNM & Method in [33] & Method in [30] & Method in [31] \\
\hline 2 & $6.30 \times 10^{-6}$ & $6.46 \times 10^{-3}$ & $2.74 \times 10^{-4}$ & $6.48 \times 10^{-4}$ \\
1 & $1.09 \times 10^{-7}$ & $1.17 \times 10^{-4}$ & $6.31 \times 10^{-6}$ & $4.39 \times 10^{-5}$ \\
$1 / 2$ & $1.53 \times 10^{-9}$ & $1.88 \times 10^{-6}$ & $1.16 \times 10^{-7}$ & $2.99 \times 10^{-6}$ \\
$1 / 4$ & $2.90 \times 10^{-11}$ & $2.96 \times 10^{-8}$ & $1.85 \times 10^{-9}$ & $1.88 \times 10^{-7}$ \\
$1 / 8$ & $5.16 \times 10^{-13}$ & $4.64 \times 10^{-10}$ & $2.92 \times 10^{-11}$ & $1.18 \times 10^{-8}$
\end{tabular}

and the analytical solution is

$$
y(t)=e^{-(\delta / 2) t} \cos \left(t \sqrt{\omega^{2}-\frac{\delta^{2}}{4}}\right),
$$

$$
\omega=1, \delta=10^{-3} \text {. }
$$

This example is solved using the Block Nyström method based on polynomial basis given in [33], Block Hybrid Trigonometrically Fitted Algorithm of order 5 given in [30], Block hybrid trigonometrically fitted Runge-Kutta-Nyström method of order 3 given in [31], and the BHFNM given in this paper. The errors obtained which are compared for different step sizes and displayed in Table 1 show that BHFNM is more accurate.

Example 7. We consider the oscillatory system that is solved in [34]

$$
\begin{aligned}
y^{\prime \prime}+\left[\begin{array}{cc}
13 & -12 \\
-12 & 13
\end{array}\right] y= & \frac{12 \varepsilon^{2}}{5}\left[\begin{array}{cc}
3 & 2 \\
-2 & 3
\end{array}\right] y^{\prime} \\
& +\varepsilon^{2}\left[\begin{array}{l}
f_{1}(t) \\
f_{2}(t)
\end{array}\right], \\
y(0)= & {\left[\begin{array}{l}
\varepsilon^{2} \\
\varepsilon^{2}
\end{array}\right], } \\
y^{\prime}(0)= & {\left[\begin{array}{c}
-4 \\
6
\end{array}\right], }
\end{aligned}
$$

with $f_{1}(t)=(36 / 5) \sin t+24 \sin 5 t$ and $f_{2}(t)=-(24 / 5) \sin t-$ $36 \sin 5 t$.

The exact solution of this system is

$$
y=\left[\begin{array}{l}
\sin t-\sin 5 t+\varepsilon \cos t \\
\sin t+\sin 5 t+\varepsilon \cos t
\end{array}\right] .
$$

This example is solved using the Block Nyström method based on polynomial basis given in [33], Block Hybrid Trigonometrically Fitted Algorithm of order 5 given in [30], Block hybrid trigonometrically fitted Runge-Kutta-Nyström method of order 3 given in [31], and the BHFNM given in this paper. The errors obtained are compared for different step sizes and displayed in Table 2 show that BHFNM is more accurate.
4.1. Application to a PDE. $L_{\infty}$ and $L_{2}$ are calculated as follows: $L_{2}=\left(\sum_{i}^{M} e_{i, j}^{2}\right)^{1 / 2}$ and $L_{\infty}=\max \left|e_{i, j}\right|, 1 \leq i \leq M$, where $e_{i, j}=\left|u_{i, j}-u\left(x_{i}, t_{j}\right)\right|, j=1, \ldots, N$.

Example 8. We consider the telegraph equation which was also solved in [33]

$$
\begin{aligned}
\frac{\partial^{2} u}{\partial t^{2}} & +2 \pi \frac{\partial u}{\partial t}+\pi^{2} u \\
& =\frac{\partial^{2} u}{\partial x^{2}}+\pi^{2} \sin \pi x(\sin \pi t+2 \cos \pi t),
\end{aligned}
$$

$$
0 \leq x \leq 1,0 \leq t \leq 1
$$

The analytical solution is given by

$$
u(x, t)=\sin \pi x \sin \pi t
$$

and the initial conditions are defined to match with the analytical solution.

This PDE is solved by first discretization the spatial variable $x$ via the finite difference method to obtain

$$
\begin{aligned}
\frac{\partial^{2} u_{i}(t)}{\partial t^{2}}= & \frac{\left(u_{i+1}(t)-2 u_{i}(t)+u_{i-1}(t)\right)}{(\Delta x)^{2}}-2 \pi \frac{\partial u_{i}(t)}{\partial t} \\
& -\pi^{2} u_{i}(t)+g_{i}(t), \quad 0<t<1,
\end{aligned}
$$

where $\Delta x=\left(x_{0}-x_{M}\right) / M, x_{i}=x_{0}+i \Delta x, i=$ $0,1, \ldots, M, u_{i}(t) \approx u\left(x_{i}, t\right), \mathbf{U}(t)=\left[u_{1}(t), \ldots, u_{M}(t)\right]^{T}$, $\mathbf{U}^{\prime}(t)=\left[u_{1}^{\prime}(t), \ldots, u_{M}^{\prime}(t)\right]^{T}, \mathbf{G}=\left[g_{1}(t)-2 \pi u_{1}^{\prime}(t)-\right.$ $\left.\pi^{2} u_{1}(t), \ldots, g_{M}(t)-2 \pi u_{M}^{\prime}(t)-\pi^{2} u_{M}(t)\right]^{T}$, and $g_{i}(t) \approx$ $g\left(x_{i}, t\right)=\pi^{2} \sin \left(\pi x_{i}\right)(\sin (\pi t)+\cos (\pi t))$, which can be written in the form

$$
\mathbf{U}^{\prime \prime}=\mathbf{f}\left(t, \mathbf{U}, \mathbf{U}^{\prime}\right)
$$

subject to the initial conditions $\mathbf{U}(\mathbf{0})=0, i=1, \ldots, M$, $\mathbf{U}^{\prime}(\mathbf{0})=\pi \sin \left(\pi x_{i}\right), i=1, \ldots, M, w=\pi$, where $\mathbf{f}\left(t, \mathbf{U}, \mathbf{U}^{\prime}\right)=$ $\mathbf{S U}+\mathbf{G}$, and $\mathbf{S}$ is a $M \times M$, matrix arising from the semidiscretized system.

This problem is solved using the BHFNM and the numerical results are displayed in Table 3 and Figure 2. The table gives $L_{\infty}$ and $L_{2}$ errors, while a comparison of the exact and numerical solutions is given in the figure plotted in 3 dimensions.

Example 9. We consider the dissipative nonlinear wave equation given in [35]

$$
\begin{aligned}
& \frac{\partial^{2} u}{\partial t^{2}} \\
& =\frac{\partial^{2} u}{\partial x^{2}}-2 u \frac{\partial u}{\partial t} \\
& \quad+\left(\pi^{2}-1-2 \sin (\pi x) \sin (t)\right)(\sin (\pi x) \cos (\pi t)), \\
& \quad 0 \leq x \leq 1,0 \leq t \leq 1,
\end{aligned}
$$


TABLE 2: Comparison of global error for Example 7 on the interval $[0,100], w=5$.

\begin{tabular}{|c|c|c|c|c|}
\hline $\mathrm{h}$ & $\operatorname{BHFNM-Error}\left(y_{1}\right)$ & $\begin{array}{l}\text { Method in [33]-Error }\left(y_{1}\right) \\
\text { Method in [30]-Error }\left(y_{1}\right) \\
\text { Method in [31]-Error }\left(y_{1}\right)\end{array}$ & $\operatorname{BHFNM-Error}\left(y_{2}\right)$ & $\begin{array}{l}\text { Method in [33]-Error }\left(y_{2}\right) \\
\text { Method in [30]-Error }\left(y_{2}\right) \\
\text { Method in [31]-Error }\left(y_{2}\right)\end{array}$ \\
\hline \multirow[t]{3}{*}{1} & $3.05 \times 10^{-3}$ & $1.97 \times 10^{0}$ & $3.04 \times 10^{-3}$ & $1.97 \times 10^{0}$ \\
\hline & & $2.92 \times 10^{-1}$ & & $2.92 \times 10^{-1}$ \\
\hline & & $2.05 \times 10^{0}$ & & $2.05 \times 10^{0}$ \\
\hline \multirow[t]{3}{*}{$1 / 2$} & $4.79 \times 10^{-5}$ & $1.24 \times 10^{-1}$ & $4.79 \times 10^{-5}$ & $1.24 \times 10^{-1}$ \\
\hline & & $3.26 \times 10^{-3}$ & & $3.26 \times 10^{-3}$ \\
\hline & & $9.07 \times 10^{-2}$ & & $9.07 \times 10^{-2}$ \\
\hline \multirow[t]{3}{*}{$1 / 4$} & $7.38 \times 10^{-7}$ & $2.30 \times 10^{-3}$ & $7.38 \times 10^{-7}$ & $2.30 \times 10^{-3}$ \\
\hline & & $4.79 \times 10^{-5}$ & & $4.79 \times 10^{-5}$ \\
\hline & & $4.95 \times 10^{-3}$ & & $4.95 \times 10^{-3}$ \\
\hline \multirow[t]{3}{*}{$1 / 8$} & $1.17 \times 10^{-8}$ & $3.76 \times 10^{-5}$ & $1.18 \times 10^{-8}$ & $3.76 \times 10^{-5}$ \\
\hline & & $7.40 \times 10^{-7}$ & & $7.40 \times 10^{-7}$ \\
\hline & & $3.00 \times 10^{-4}$ & & $3.00 \times 10^{-4}$ \\
\hline \multirow[t]{3}{*}{$1 / 16$} & $1.80 \times 10^{-10}$ & $5.93 \times 10^{-7}$ & $1.80 \times 10^{-10}$ & $5.93 \times 10^{-7}$ \\
\hline & & $1.19 \times 10^{-8}$ & & $1.20 \times 10^{-8}$ \\
\hline & & $1.86 \times 10^{-5}$ & & $1.86 \times 10^{-5}$ \\
\hline \multirow[t]{3}{*}{$1 / 32$} & $2.92 \times 10^{-12}$ & $9.38 \times 10^{-9}$ & $3.06 \times 10^{-12}$ & $9.38 \times 10^{-9}$ \\
\hline & & $1.80 \times 10^{-10}$ & & $1.80 \times 10^{-10}$ \\
\hline & & $1.16 \times 10^{-6}$ & & $1.16 \times 10^{-6}$ \\
\hline
\end{tabular}

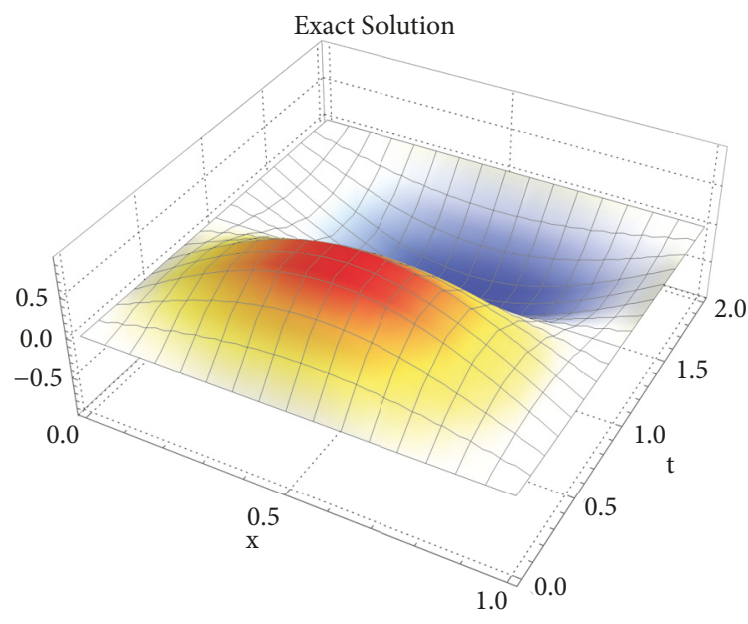

(a) Exact solution

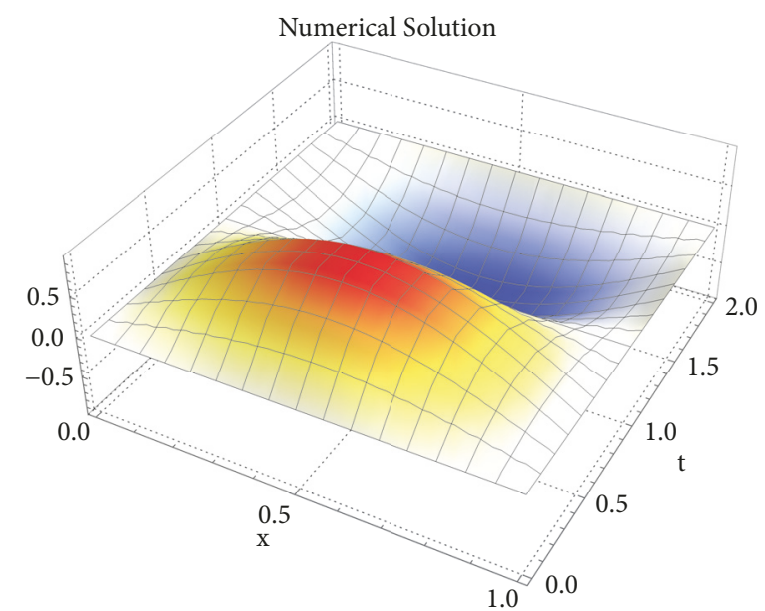

(b) Numerical solution

Figure 2: 3D plots of exact solution (a) and numerical solution (b) for Example 8 up to $t=2, M=20$, and $N=10$.

with initial conditions

$$
\begin{aligned}
& u(x, 0)=\sin (\pi x), \\
& u_{t}(x, 0)=0 .
\end{aligned}
$$

The analytical solution is given by

$$
u(x, t)=\sin (\pi x) \cos (t)
$$

and the initial conditions are defined to match with the analytical solution.
This PDE is solved by first discretization the spatial variable $x$ via the finite difference method to obtain

$$
\begin{aligned}
\frac{\partial^{2} u_{i}(t)}{\partial t^{2}}= & \frac{\left(u_{i+1}(t)-2 u_{i}(t)+u_{i-1}(t)\right)}{(\Delta x)^{2}}-2 \pi \frac{\partial u_{i}(t)}{\partial t} \\
& -\pi^{2} u_{i}(t)+g_{i}(t), \quad 0<t<1,
\end{aligned}
$$

where $\Delta x=\left(x_{0}-x_{M}\right) / M, x_{i}=x_{0}+i \Delta x, i=0,1, \ldots, M$, $u_{i}(t) \approx u\left(x_{i}, t\right), \mathbf{U}(t)=\left[u_{1}(t), \ldots, u_{M}(t)\right]^{T}, \mathbf{U}^{\prime}(t)=$ $\left[u_{1}^{\prime}(t), \ldots, u_{M}^{\prime}(t)\right]^{T}, \mathbf{G}=\left[g_{1}(t)-2 u_{1}(t) u_{1}^{\prime}(t), \ldots, g_{M}(t)-\right.$ $\left.2 u_{M}(t) u_{M}^{\prime}(t)\right]^{T}$, and $g_{i}(t) \approx g\left(x_{i}, t\right)=\left(\pi^{2}-1-\right.$ 


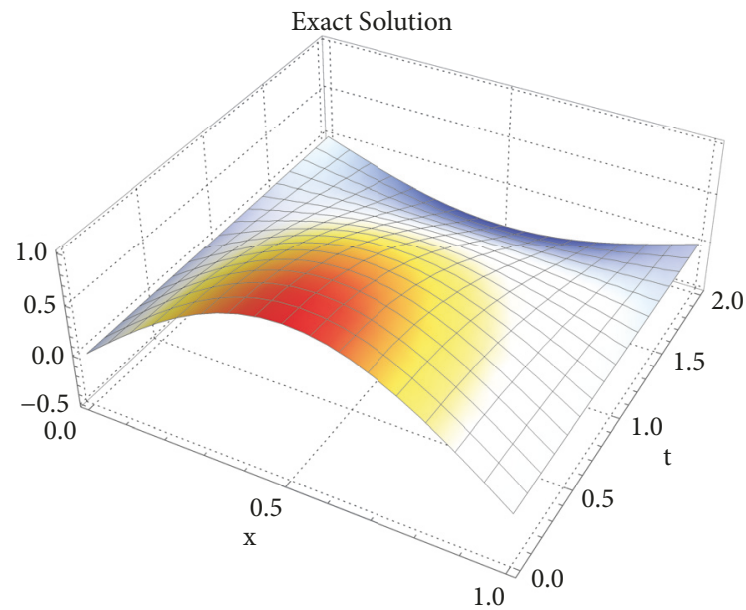

(a) Exact solution

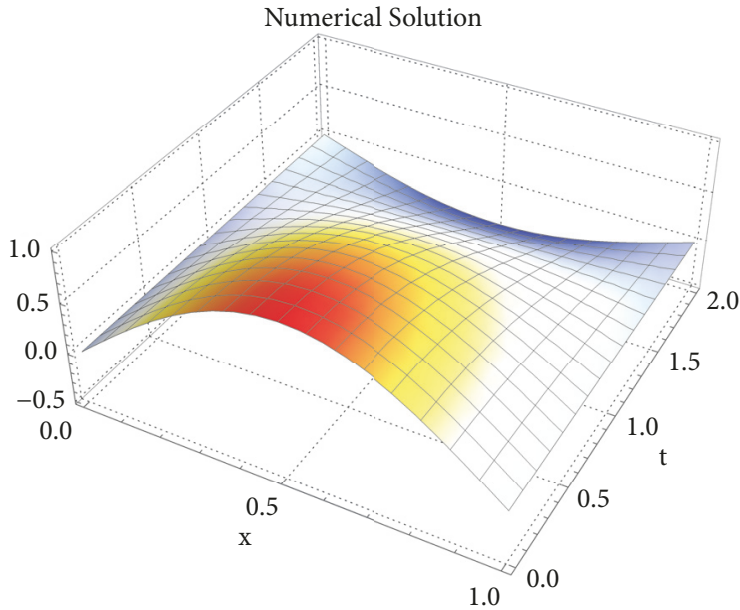

(b) Numerical solution

FIGURE 3: 3D plots of exact solution (a) and numerical solution (b) for Example 9 up to $t=2, M=20$, and $N=10$.

TABLE 3: $L_{\infty}$ and $L_{2}$ errors for Example 8, for $M=20$ and $N=10$ at different times.

\begin{tabular}{ccc}
\hline $\mathrm{T}$ & $L_{\infty}$ & $L_{2}$ \\
\hline 1 & $7.86979 \times 10^{-4}$ & $2.48865 \times 10^{-3}$ \\
2 & $8.20935 \times 10^{-4}$ & $2.59602 \times 10^{-3}$ \\
3 & $8.22400 \times 10^{-4}$ & $2.60066 \times 10^{-3}$ \\
4 & $8.22463 \times 10^{-4}$ & $2.60086 \times 10^{-3}$ \\
5 & $8.22467 \times 10^{-4}$ & $2.60087 \times 10^{-3}$ \\
\hline
\end{tabular}

$\left.2 \sin \left(\pi x_{i}\right) \sin (t)\right)\left(\sin \left(\pi x_{i}\right) \cos (\pi t)\right)$, which can be written in the form

$$
\mathbf{U}^{\prime \prime}=\mathbf{f}\left(t, \mathbf{U}, \mathbf{U}^{\prime}\right),
$$

subject to the initial conditions $\mathbf{U}(\mathbf{0})=\sin \left(\pi x_{i}\right), i=1, \ldots, M$, $\mathbf{U}^{\prime}(\mathbf{0})=0, i=1, \ldots, M$, where $\mathbf{f}\left(t, \mathbf{U}, \mathbf{U}^{\prime}\right)=\mathbf{S} \mathbf{U}+\mathbf{G}$, and $\mathbf{S}$ is a $M \times M, w=\pi$, matrix arising from the semidiscretized system.

This problem is solved using the BHFNM and the numerical results are displayed in Table 4 and Figure 3. The table gives $L_{\infty}$ and $L_{2}$ errors, while a comparison of the exact and numerical solutions is given in the figure plotted in 3 dimensions.

Example 10. We consider the dissipative nonlinear wave equation given in [35]

$$
\begin{aligned}
\frac{\partial^{2} u}{\partial t^{2}}= & \frac{\partial^{2} u}{\partial x^{2}}+\Upsilon\left(u^{2}-1\right) \frac{\partial u}{\partial t} \\
& +\left(\pi^{2}+\Upsilon^{2} e^{-2 \Upsilon t} \sin ^{2}(\pi x) e^{-\Upsilon t}\right) \sin (\pi x), \\
& \quad 0 \leq x \leq 1,0 \leq t \leq 1,
\end{aligned}
$$

with initial conditions

$$
\begin{aligned}
u(x, 0) & =\sin (\pi x), \\
u_{t}(x, 0) & =-\Upsilon \sin (\pi x) .
\end{aligned}
$$

The analytical solution is given by

$$
u(x, t)=e^{-\Upsilon t} \sin (\pi x)
$$

and the initial conditions are defined to match with the analytical solution.

This PDE is solved by first discretization the spatial variable $x$ via the finite difference method to obtain

$$
\begin{aligned}
\frac{\partial^{2} u_{i}(t)}{\partial t^{2}}= & \frac{\left(u_{i+1}(t)-2 u_{i}(t)+u_{i-1}(t)\right)}{(\Delta x)^{2}} \\
& +\Upsilon\left(u^{2}-1\right) \frac{\partial u_{i}(t)}{\partial t}+g_{i}(t), \quad 0<t<1,
\end{aligned}
$$

where $\Delta x=\left(x_{0}-x_{M}\right) / M, x_{i}=x_{0}+i \Delta x, i=$ $0,1, \ldots, M, u_{i}(t) \approx u\left(x_{i}, t\right), \mathbf{U}(t)=\left[u_{1}(t), \ldots, u_{M}(t)\right]^{T}$, $\mathbf{U}^{\prime}(t)=\left[u_{1}^{\prime}(t), \ldots, u_{M}^{\prime}(t)\right]^{T}, \mathbf{G}=\left[g_{1}(t)-\Upsilon\left(u_{1}(t)^{2}-\right.\right.$ 1) $\left.u_{1}^{\prime}(t), \ldots, g_{M}(t)-\Upsilon\left(u_{M}(t)^{2}-1\right) u_{M}^{\prime}(t)\right]^{T}$, and $g_{i}(t) \approx$ $g\left(x_{i}, t\right)=\left(\pi^{2}+\Upsilon^{2} e^{-2 Y t} \sin ^{2}\left(\pi x_{i}\right) e^{-\Upsilon t}\right) \sin \left(\pi x_{i}\right)$, which can be written in the form

$$
\mathbf{U}^{\prime \prime}=\mathbf{f}\left(t, \mathbf{U}, \mathbf{U}^{\prime}\right)
$$

subject to the initial conditions $\mathbf{U}(\mathbf{0})=\sin \left(\pi x_{i}\right), i=1, \ldots, M$, $\mathbf{U}^{\prime}(\mathbf{0})=-\Upsilon \sin \left(\pi x_{i}\right), i=1, \ldots, M$, where $\mathbf{f}\left(t, \mathbf{U}, \mathbf{U}^{\prime}\right)=$ $\mathbf{S U}+\mathbf{G}$, and $\mathbf{S}$ is a $M \times M, w=\pi$, matrix arising from the semidiscretized system.

This problem is solved using the BHFNM and the numerical results are displayed in Table 5 and Figure 4 . The table gives $L_{\infty}$ and $L_{2}$ errors, while a comparison of the exact and numerical solutions are given in the figure plotted in 3 dimensions.

4.2. Estimating the Frequency. Some techniques for estimating the frequency are given in [24, 36-39]. In the spirit of [36], where the technique for estimating the frequency was to determine the roots of the polynomial associated with the 


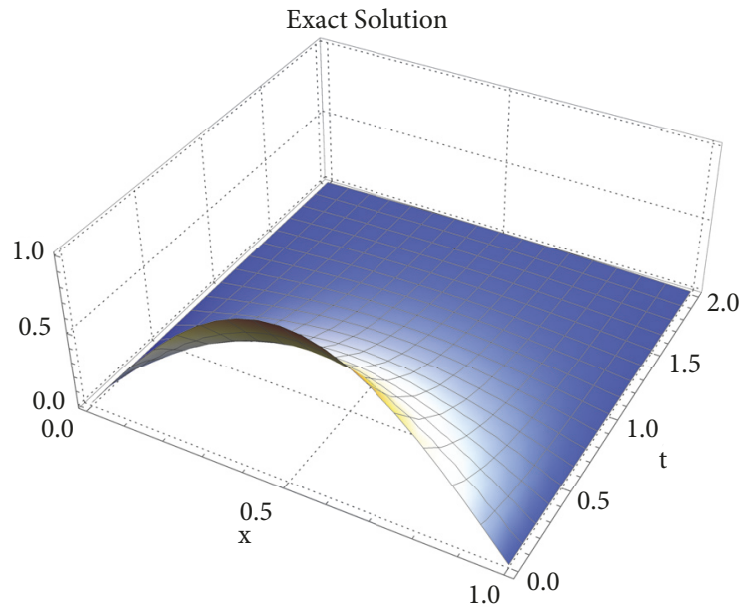

(a) Exact solution

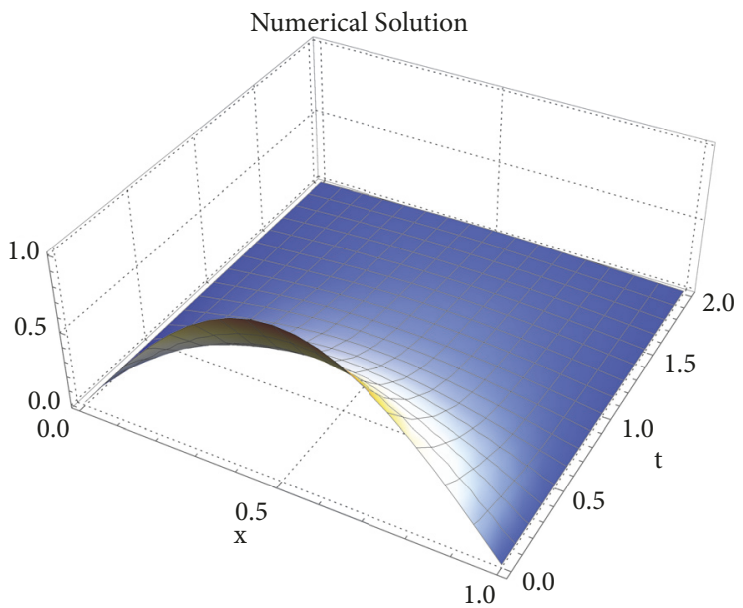

(b) Numerical solution

FIGURE 4: 3D plots of exact solution (a) and numerical solution (b) for Example 10 up to $t=2, M=20, N=10$, and $\Upsilon=2$.

TABLE 4: $L_{\infty}$ and $L_{2}$ errors for Example 9, for $M=N=10$ at different times.

\begin{tabular}{lcc}
\hline $\mathrm{T}$ & $L_{\infty}$ & $L_{2}$ \\
\hline 1 & $1.12220 \times 10^{-2}$ & $1.74162 \times 10^{-2}$ \\
2 & $8.62553 \times 10^{-3}$ & $1.46484 \times 10^{-2}$ \\
3 & $6.58683 \times 10^{-3}$ & $1.52150 \times 10^{-2}$ \\
4 & $6.97169 \times 10^{-3}$ & $1.46843 \times 10^{-2}$ \\
5 & $7.99197 \times 10^{-3}$ & $1.74162 \times 10^{-2}$ \\
\hline
\end{tabular}

TABle 5: $L_{\infty}$ and $L_{2}$ errors for Example 10, for $M=N=10$ and $\Upsilon=1$ at different times.

\begin{tabular}{ccc}
\hline $\mathrm{T}$ & $L_{\infty}$ & $L_{2}$ \\
\hline 1 & $8.26147 \times 10^{-3}$ & $1.59350 \times 10^{-2}$ \\
2 & $7.51186 \times 10^{-3}$ & $1.54297 \times 10^{-2}$ \\
3 & $3.78627 \times 10^{-3}$ & $7.50972 \times 10^{-2}$ \\
4 & $2.87725 \times 10^{-3}$ & $5.80621 \times 10^{-2}$ \\
5 & $3.78271 \times 10^{-3}$ & $6.09587 \times 10^{-2}$ \\
\hline
\end{tabular}

LTE, we rewrite the principal terms given in (20) and (21) as follows:

$$
\begin{aligned}
& \varpi_{j} \\
& =\left\{\begin{array}{l}
\varrho_{j}\left(w_{n}^{2} y^{(5)}\left(x_{n}\right)+y^{(7)}\left(x_{n}\right)\right)=\varrho_{j} D^{5}\left(w_{n}^{2}+D^{2}\right) y\left(x_{n}\right), \\
\varrho_{8}\left(w_{n}^{2} y^{(6)}\left(x_{n}\right)+y^{(8)}\left(x_{n}\right)\right)=\varrho_{8} D^{6}\left(w_{n}^{2}+D^{2}\right) y\left(x_{n}\right) .
\end{array}\right.
\end{aligned}
$$

where $\varrho_{j}, j=1, \ldots, 8$, are constants, $y^{(j)}=d^{j} y / d x^{j}$, the positive $j$ represents the $j^{\text {th }}$ derivative, and $D=d / d x$ is a linear differential operator and therefore invertible, which facilitates the simplification of $\omega_{j}$. We estimate $w_{n}$ by setting any member of $\widehat{\omega}_{j}=0$ and solving for $w_{n}$ to obtain two possible solutions of $w_{n}$ as follows:

$$
w_{n}= \pm \sqrt{-\frac{D^{7} y\left(x_{n}\right)}{D^{5} y\left(x_{n}\right)}}= \pm \sqrt{-\frac{D^{2} y\left(x_{n}\right)}{y\left(x_{n}\right)}} .
$$

TABLE 6: Comparison of global errors for Example 6 on [0,100], $w=$ $1, w=w_{n}$, and $w=w_{0}$.

\begin{tabular}{lccc}
\hline $\mathrm{h}$ & BHFNM $(w=1)$ & BHFNM $\left(w=w_{n}\right)$ & BHFNM $\left(w=w_{0}\right)$ \\
\hline 2 & $6.30 \times 10^{-6}$ & $6.28 \times 10^{-6}$ & $6.30 \times 10^{-6}$ \\
1 & $1.09 \times 10^{-7}$ & $5.35 \times 10^{-7}$ & $1.09 \times 10^{-7}$ \\
$1 / 2$ & $1.53 \times 10^{-9}$ & $4.83 \times 10^{-9}$ & $1.45 \times 10^{-9}$ \\
$1 / 4$ & $2.90 \times 10^{-11}$ & $5.74 \times 10^{-11}$ & $2.90 \times 10^{-11}$ \\
$1 / 8$ & $5.16 \times 10^{-13}$ & $8.29 \times 10^{-13}$ & $4.41 \times 10^{-13}$ \\
\hline
\end{tabular}

This estimate has been tested on Example 6 on [0,100], for $w=w_{n}$, where $w_{n}=\sqrt{\omega^{2}+\delta y^{\prime}\left(x_{n}\right) / y\left(x_{n}\right)}$.

We observe that when $w=w_{n}$ was used, an appropriate estimate for the parameter was provided at each step, in which case the computational time was increased and results were slightly less accurate than those given by the exact frequency, $w=1$. We then noticed that, for this example, the results produced by setting $w=w_{0}$ were similar to those given by $w=1$. Therefore, for this example, the appropriate choice for the frequency is $w=w_{0}$. We do not claim that this approach will work in all cases; nevertheless, we will rigorously conduct research on this topic in our future work. Details of the numerical results are given in Table 6.

\section{Conclusion}

We have presented a BHFNM with coefficients that depend on a fixed frequency and the stepsize and that is implemented in a block-by-block fashion, which does not suffer from the disadvantages of requiring starting values and predictors inherent to predictor-corrector methods. It can be used to solve general second-order IVP, in particular, those that arise from the semidiscretization of hyperbolic PDE. Numerical studies have been presented to establish accuracy of the method. Since the exact value of the parameter $w$ is not always available, we have attempted to estimate an appropriate value 
of the parameter $w$, in the spirit of [36], where the technique for estimating the frequency was to determine the roots of the polynomial associated with the LTE. We do not claim that this approach will work for all cases; hence we are determined to conduct rigorous research on this topic in our future work. We also note that the methods presented were not designed to incorporate a variable stepsize implementation, since we did not consider additional methods which are required to provide the error estimates as we proceed on the interval of interest. Nevertheless, our future research will include a variable stepsize implementation of the methods.

\section{Data Availability}

All references including data used to support the findings of this study are included within the article.

\section{Conflicts of Interest}

The authors declare that there are no conflicts of interest regarding the publication of this paper.

\section{References}

[1] J. D. Lambert, Numerical Methods for Ordinary Differential Systems. The Initial Value Problem, John Wiley \& Sons, New York, NY, USA, 1993.

[2] J. D. Lambert, Computational Methods in Ordinary Differential Equations, John Wiley \& Sons, New York, NY, USA, 1973.

[3] E. Hairer and G. Wanner, Solving Ordinary Differential Equations II, Springer, New York, NY, USA, 1996.

[4] E. Hairer, "A one-step method of order 10 for $y^{\prime \prime}=f(x, y)$," IMA Journal of Numerical Analysis (IMAJNA), vol. 2, no. 1, pp. 83-94, 1982.

[5] L. Brugnano and D. Trigiante, Solving ODEs By Multistep Initial and Boundary Value Methods, Gordon \& Breach, Amsterdam, 1998.

[6] L. Brugnano and D. Trigiante, "Block implicit methods for ODEs," in Recent Trends in Numerical Analysis, D. Trigiante, Ed., pp. 81-105, Nova Science Publishers, New York, NY, USA, 2001.

[7] E. Hairer, S. P. Nørsett, and G. Wanner, Solving Ordinary Differential Equations I: Nonstiff Problems, vol. 8 of Springer Series in Computational Mathematics, Springer, Berlin, Heidelberg, Germany, 1993.

[8] T. E. Simos, "An exponentially-fitted Runge-Kutta method for the numerical integration of initial-value problems with periodic or oscillating solutions," Computer Physics Communications, vol. 115, no. 1, pp. 1-8, 1998.

[9] J. D. Lambert and I. A. Watson, "Symmetric multistep methods for periodic initial value problems," Journal of the Institute of Mathematics and Its Applications, vol. 18, no. 2, pp. 189-202, 1976.

[10] E. H. Twizell and A. Q. M. Khaliq, "Multiderivative methods for periodic initial value problems," SIAM Journal on Numerical Analysis, vol. 21, no. 1, pp. 111-122, 1984.

[11] J. Vigo-Aguiar and H. Ramos, "Dissipative Chebyshev exponential-fitted methods for numerical solution of secondorder differential equations," Journal of Computational and Applied Mathematics, vol. 158, no. 1, pp. 187-211, 2003.
[12] D. O. Awoyemi, "A new sixth-order algorithm for general second order ordinary differential equation," International Journal of Computer Mathematics, vol. 77, no. 1, pp. 117-124, 2001.

[13] M. M. Chawla and S. R. Sharma, "Families of three-stage third order Runge-Kutta-Nystrom methods for $y^{\prime \prime}=f\left(x, y, y^{\prime}\right)$," The Journal of the Australian Mathematical Society, vol. 26, no. 3, pp. 375-386, 1985.

[14] S. M. Mahmoud and M. S. Osman, "On a class of splinecollocation methods for solving second-order initial-value problems," International Journal of Computer Mathematics, vol. 86, no. 4, pp. 616-630, 2009.

[15] J. M. Franco, "Runge-Kutta-Nyström methods adapted to the numerical integration of perturbed oscillators," Computer Physics Communications, vol. 147, no. 3, pp. 770-787, 2002.

[16] S. N. Jator, "Implicit third derivative Runge-Kutta-Nyström method with trigonometric coefficients," Numerical Algorithms, vol. 70, no. 1, pp. 133-150, 2015.

[17] S. N. Jator, "A continuous two-step method of order 8 with a block extension for $y^{\prime \prime}=f\left(x, y, y^{\prime}\right)$," Applied Mathematics and Computation, vol. 219, no. 3, pp. 781-791, 2012.

[18] J. P. Coleman and S. C. Duxbury, "Mixed collocation methods for $y^{\prime \prime}=f(x, y)$," Journal of Computational and Applied Mathematics, vol. 126, no. 1-2, pp. 47-75, 2000.

[19] J. P. Coleman and L. G. Ixaru, " $P$-stability and exponentialfitting methods for $y^{\prime \prime}=f(x, y)$," IMA Journal of Numerical Analysis (IMAJNA), vol. 16, no. 2, pp. 179-199, 1996.

[20] T. E. Simos, "Dissipative trigonometrically-fitted methods for second order IVPs with oscillating solution," International Journal of Modern Physics C, vol. 13, no. 10, pp. 1333-1345, 2002.

[21] G. Vanden, L. Gr. Ixaru, and M. van Daele, "Optimal implicit exponentially-fitted Runge-Kutta," Computer Physics Communications, vol. 140, pp. 346-357, 2001.

[22] Y. Fang, Y. Song, and $\mathrm{X}$. Wu, "A robust trigonometrically fitted embedded pair for perturbed oscillators," Journal of Computational and Applied Mathematics, vol. 225, no. 2, pp. 347-355, 2009.

[23] H. S. Nguyen, R. B. Sidje, and N. H. Cong, "Analysis of trigonometric implicit Runge-Kutta methods," Journal of Computational and Applied Mathematics, vol. 198, no. 1, pp. 187-207, 2007.

[24] H. Ramos and J. Vigo-Aguiar, "A trigonometrically-fitted method with two frequencies, one for the solution and another one for the derivative," Computer Physics Communications, vol. 185, no. 4, pp. 1230-1236, 2014.

[25] J. M. Franco and I. Gómez, "Trigonometrically fitted nonlinear two-step methods for solving second order oscillatory IVPs," Applied Mathematics and Computation, vol. 232, pp. 643-657, 2014.

[26] K. Ozawa, "A functionally fitted three-stage explicit singly diagonally implicit Runge-Kutta method," Japan Journal of Industrial and Applied Mathematics, vol. 22, no. 3, pp. 403-427, 2005.

[27] S. N. Jator, S. Swindell, and R. French, "Trigonometrically fitted block Numerov type method for $y^{\prime \prime}=f\left(x, y, y^{\prime}\right)$," Numerical Algorithms, vol. 62, no. 1, pp. 13-26, 2013.

[28] F. F. Ngwane and S. N. Jator, "Block hybrid method using trigonometric basis for initial value problems with oscillating solutions," Numerical Algorithms, vol. 63, no. 4, pp. 713-725, 2013.

[29] S. N. Jator, "Block third derivative method based on trigonometric polynomials for periodic initial-value problems," Afrika Matematika, pp. 1-13, 2015. 
[30] F. F. Ngwane and S. N. Jator, "Solving the telegraph and oscillatory differential equations by a block hybrid trigonometrically fitted algorithm," International Journal of Differential Equations, vol. 2015, Article ID 347864, 15 pages, 2015.

[31] F. F. Ngwane and S. N. Jator, "A trigonometrically fitted block method for solving oscillatory second-order initial value problems and hamiltonian systems," International Journal of Differential Equations, vol. 2017, Article ID 9293530, 14 pages, 2017.

[32] J. B. Keiper and C. W. Gear, "The analysis of generalized backward difference formula methods applied to Hessenberg form differential-algebraic equations," SIAM Journal on Numerical Analysis, vol. 28, no. 3, pp. 833-858, 1991.

[33] S. N. Jator and H. B. Oladejo, "Block Nyström method for singular differential equations of the Lane-Emden type and problems with highly oscillatory solutions," International Journal of Applied and Computational Mathematics, vol. 3, no. suppl. 1, pp. S1385-S1402, 2017.

[34] K. Liu and X. Wu, "Multidimensional ARKN methods for general oscillatory second-order initial value problems," Computer Physics Communications, vol. 185, no. 7, pp. 1999-2007, 2014.

[35] R. Jiwari, "Lagrange interpolation and modified cubic B-spline differential quadrature methods for solving hyperbolic partial differential equations with Dirichlet and Neumann boundary conditions," Computer Physics Communications, vol. 193, pp. 5565, 2015.

[36] R. D’Ambrosio, E. Esposito, and B. Paternoster, "Parameter estimation in exponentially fitted hybrid methods for second order differential problems," Journal of Mathematical Chemistry, vol. 50, no. 1, pp. 155-168, 2012.

[37] R. D’Ambrosio, E. Esposito, and B. Paternoster, “Exponentially fitted two-step Runge-Kutta methods: construction and parameter selection," Applied Mathematics and Computation, vol. 218, no. 14, pp. 7468-7480, 2012.

[38] L. Gr. Ixaru, G. Vanden Berghe, and H. De Meyer, "Frequency evaluation in exponential fitting multistep algorithms for ODEs," Journal of Computational and Applied Mathematics, vol. 140, pp. 423-434, 2002.

[39] H. Ramos and J. Vigo-Aguiar, "On the frequency choice in trigonometrically fitted methods," Applied Mathematics Letters, vol. 23, no. 11, pp. 1378-1381, 2010. 


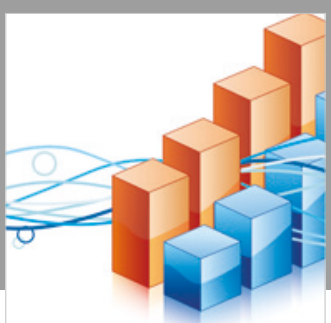

Advances in

Operations Research

\section{-n-m}
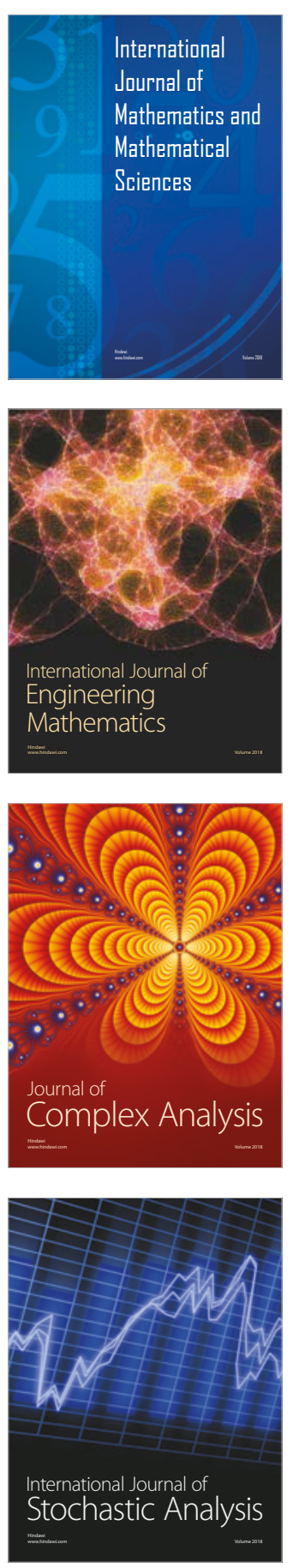
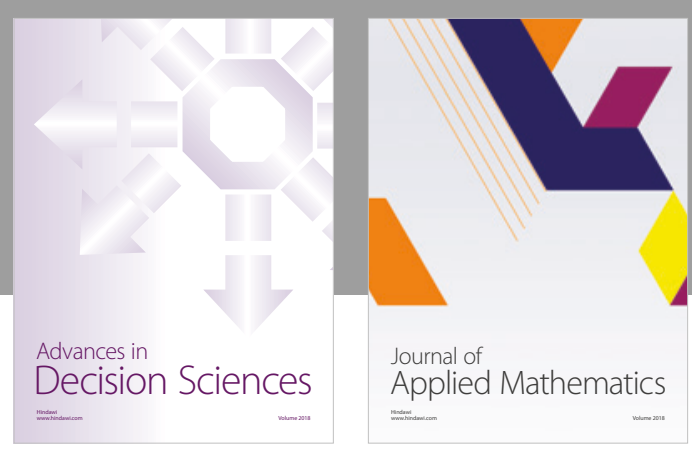

Journal of

Applied Mathematics
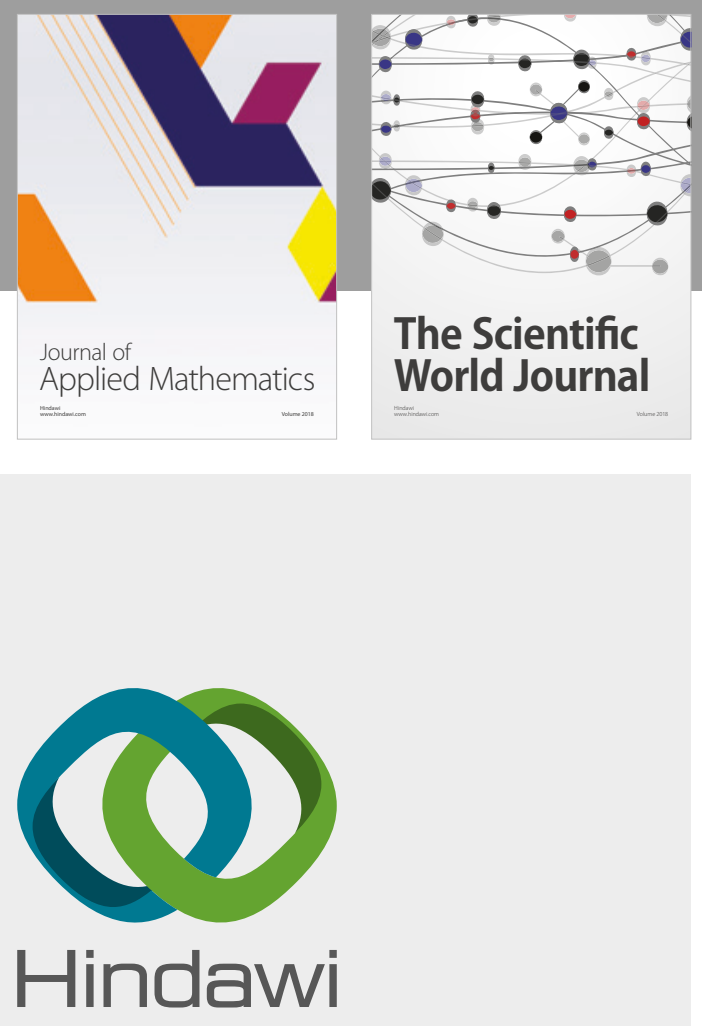

Submit your manuscripts at

www.hindawi.com

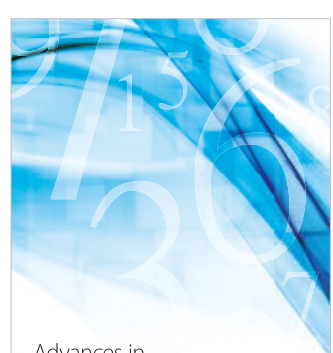

Advances in
Numerical Analysis
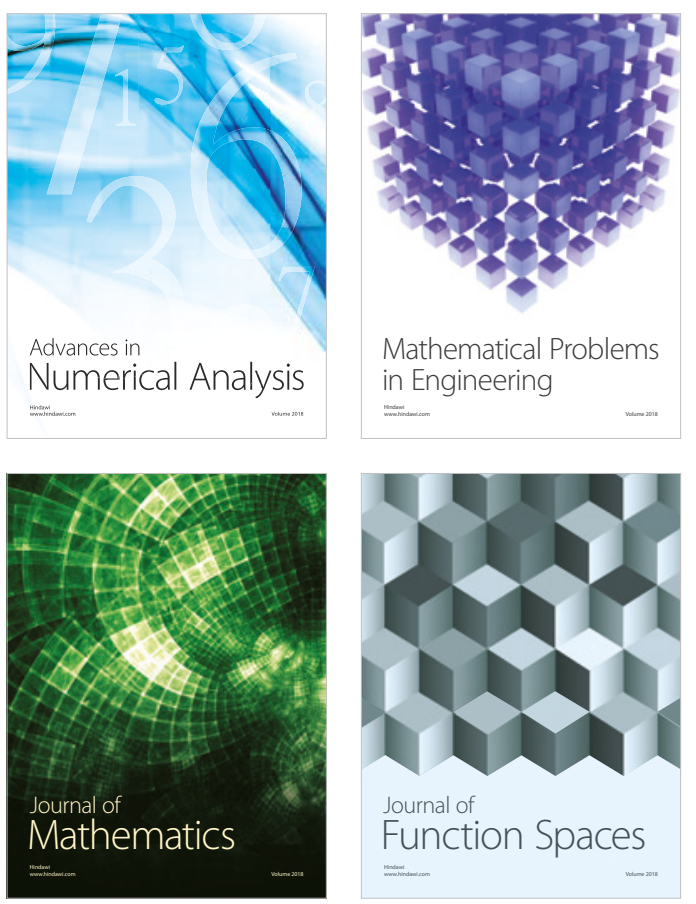

Mathematical Problems in Engineering

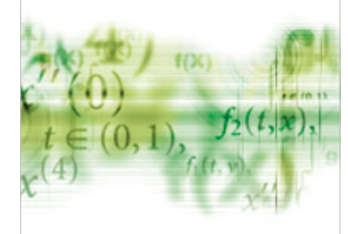

International Journal of

Differential Equations

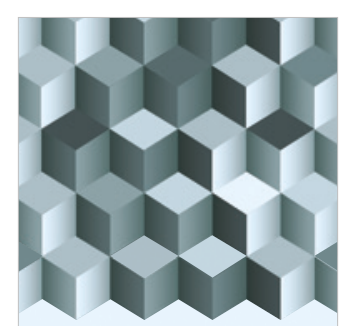

Journal of

Function Spaces

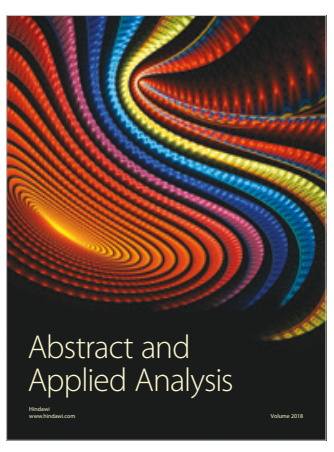

The Scientific

World Journal

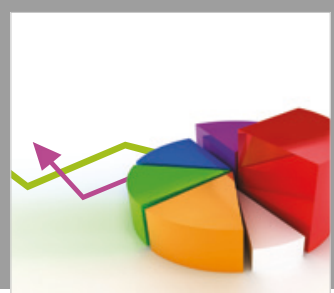

Journal of

Probability and Statistics
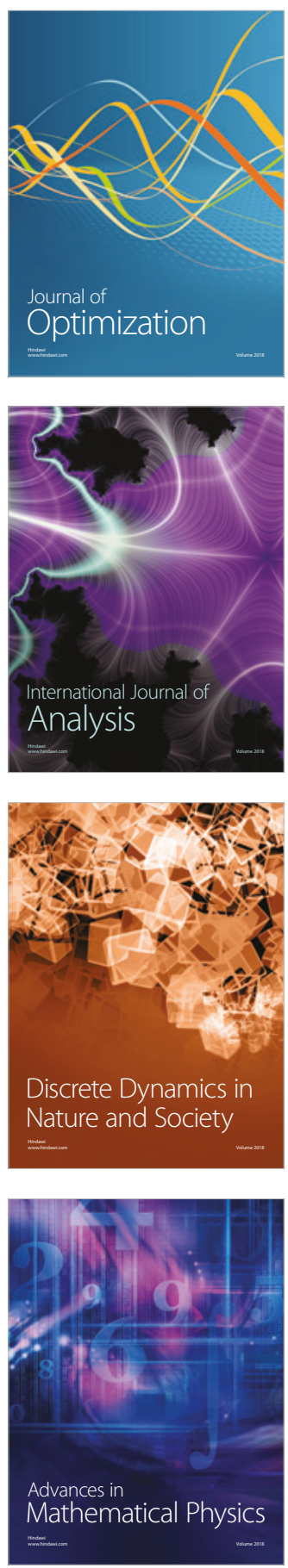